OPEN ACCESS

Edited by:

Catherine Disselhorst-Klug,

RWTH Aachen University, Germany

Reviewed by:

Roberto Merletti,

Politecnico di Torino, Italy Andrea Merlo,

Ospedale Privato Accreditato Sol et Salus, Italy

*Correspondence: Rakesh Pilkar

rpilkar@kesslerfoundation.org

${ }^{\dagger}$ Present address: Rakesh Pilkar,

Center for Mobility and Rehabilitation Engineering Research, Kessler Foundation, West Orange, NJ, United States

Specialty section:

This article was submitted to Neurorehabilitation,

a section of the journal

Frontiers in Neurology

Received: 30 June 2020 Accepted: 19 November 2020 Published: 18 December 2020

Citation:

Pilkar R, Momeni K, Ramanujam A, Ravi M, Garbarini $E$ and Forrest GF (2020) Use of Surface EMG in Clinical Rehabilitation of Individuals With SCl: Barriers and Future Considerations. Front. Neurol. 11:578559. doi: 10.3389/fneur.2020.578559

\section{Use of Surface EMG in Clinical Rehabilitation of Individuals With SCl: Barriers and Future Considerations}

\author{
Rakesh Pilkar ${ }^{1,2 \star \dagger}$, Kamyar Momeni ${ }^{2,3}$, Arvind Ramanujam ${ }^{4}$, Manikandan Ravi ${ }^{3}$, \\ Erica Garbarini ${ }^{3}$ and Gail F. Forrest ${ }^{1,2,3}$ \\ ${ }^{1}$ Center for Mobility and Rehabilitation Engineering Research, Kessler Foundation, West Orange, NJ, United States, \\ ${ }^{2}$ Department of Physical Medicine and Rehabilitation, Rutgers - New Jersey Medical School, Newark, NJ, United States, \\ ${ }^{3}$ Tim and Caroline Reynolds Center for Spinal Stimulation, Kessler Foundation, West Orange, NJ, United States, ${ }^{4}$ Koneksa \\ Health, New York, NY, United States
}

Surface electromyography (SEMG) is a widely used technology in rehabilitation research and provides quantifiable information on the myoelectric output of a muscle. In this perspective, we discuss the barriers which have restricted the wide-spread use of sEMG in clinical rehabilitation of individuals with spinal cord injury (SCI). One of the major obstacles is integrating the time-consuming aspects of $\mathrm{SEMG}$ in the already demanding schedule of physical therapists, occupational therapists, and other clinicians. From the clinicians' perspective, the lack of confidence to use sEMG technology is also apparent due to their limited exposure to the SEMG technology and possibly limited mathematical foundation through educational and professional curricula. Several technical challenges include the limited technology-transfer of ever-evolving knowledge from sEMG research into the off-the-shelf EMG systems, lack of demand from the clinicians for systems with advanced features, lack of user-friendly intuitive interfaces, and the need for a multidisciplinary approach for accurate handling and interpretation of data. We also discuss the challenges in the application and interpretation of sEMG that are specific to $\mathrm{SCl}$, which are characterized by non-standardized approaches in recording and interpretation of EMGs due to the physiological and structural state of the spinal cord. Addressing the current barriers will require a collaborative, interdisciplinary, and unified approach. The most relevant steps could include enhancing user-experience for students pursuing clinical education through revised curricula through sEMG-based case studies/projects, hands-on involvement in the research, and formation of a common platform for clinicians and technicians for self-education and knowledge share.

Keywords: spinal cord injury (SCI), electromyography (EMG), EMG barriers, biomedical signal processing, clinical rehabilitation, high-density EMG

\section{INTRODUCTION}

The current state-of-the-art rehabilitation for individuals with spinal cord injuries (SCI) utilizes technologies such as neuromodulation using exoskeleton robotics, functional electrical stimulation (FES), treadmill training with and without body-weight support (BWS) in addition to the traditional exercise-based rehabilitation. The recent developments in neurorehabilitation research 
and technologies have resulted in a shift in focus toward the recovery of function through high intensity repetitive training after SCI (1). Some of the technologies such as epidural or transcutaneous spinal stimulation, robotic exoskeletons are currently under investigation while techniques such as FES (e.g., FES cycling, rowing) (2) and treadmill training using BWS (3) are commonly used in clinics to assist with the functional tasks such as respiration, mobility, hand function, metabolism, bladder, bowel or sexual function $(2,4,5)$. Irrespective of the intervention approach used, the functional status as well as the evolutions of motor impairments and motor recovery are often tracked by visual and manual assessments in the clinic. To date, the primary method for evaluating the motor function for SCI is the American Spinal Injury Association Impairment Scale (AIS), which tests manual muscle strength in five key muscles in each limb and examines sensory function (6). Although easy to perform, such approaches are subjective and not sensitive to understanding the changes at the neuromuscular levels. Particularly for the interventions that target the neuromuscular mechanisms via application of electrical stimulation to the nerves, or peripheral musculature, the objective and quantifiable information on the myoelectric output of targeted muscles is highly relevant. For instance, when a clinician uses FES -the technique that involves the application of electrical current to the neuromuscular junction and cause contractions in paralyzed muscles (7)- it is clinically desirable to evaluate the resultant myoelectric output of the stimulated muscle. The questions such as "is the stimulation intensity sufficient to induce the desired contraction for the targeted movement?" "is the stimulation causing the targeted muscle to fatigue?" or "are the selected parameters appropriate for the patient to perform the desired task?" become highly relevant to the clinician to deliver patient-specific and effective interventions. Such questions are highly significant for any intervention that targets mobility and motor rehabilitation.

Surface electromyography (sEMG), a non-invasive technique for assessing the myoelectric output of a muscle, can provide objective answers to these significant questions. SEMG has shown great promise in neurorehabilitation research and has been a widely-utilized tool to assess neuromuscular outcomes in research (8). However, the application of sEMG in a clinical environment has been limited (9). The clinicians' perspectives on the use of sEMG have reported several barriers including limited time and resources, clinically inapplicable sEMG system features and the majority of clinicians' lack of training and/or confidence in utilization of sEMG technology $(10,11)$. In the domain of the SCI population, in addition to the aforementioned challenges of using sEMG in the clinic, severely impaired physiological and structural state of the spinal cord after SCI (compared to other pathologies such as stroke, traumatic brain injury, multiple sclerosis, etc.) further limits sEMG usage to provide time-efficient, meaningful interpretations. In this perspective report, we discuss these barriers and the directions toward overcoming these limitations that hinder the widespread use of sEMG technology in the clinical rehabilitation of individuals with SCI.

\section{BARRIERS IN THE USE OF SEMG IN SCI NEUROREHABILITATION \\ General Barriers to Use sEMG in a Clinical Setting}

Several barriers can be identified that restrict the adoption of sEMG technology in a clinical environment.

\section{Lack of Information at Motor Unit (MU) Level}

Needle EMG (nEMG) and fine wire EMG (fwEMG) are the invasive forms of EMG for accessing neurophysiological attributes of neuromuscular diseases. However, the invasiveness, discomfort, and limited applicability of these techniques on multiple muscles during dynamic tasks limit their use in the clinic. Nonetheless, nEMG still is gold standard for clinical diagnosis of nerve and muscle pathologies and preferred over non-invasive sEMG (12) for neurophysiological applications. This is because of the limited spatial resolution of sEMG that results in poor fidelity recordings of high-frequency signals (e.g., polyphasic potentials, fibrillation potentials, and positive sharp waves) (12). In addition, the electrical cross-talk between two or more neighboring muscles restricts the sEMG to identify the origin of the electrical signal when these muscles are active simultaneously (12). Further, the sEMG recorded from a muscle does not yield a non-ambiguous extraction of single MU information. As a result, the report of the therapeutics and technology assessment subcommittee of the American Academy of Neurology reported the sEMG technique unusable for clinical neurophysiological purposes (13). While the bipolar sEMG is used to measure muscle activations, the advent of highdensity surface EMG (HDEMG) has made the extraction of MU features possible (14-16). Availability of such a sensitive tool is even more significant for individuals with clinically diagnosed motor and sensory complete SCI who do not have intact reflexes and who may still have intact neuronal axons across the injury lesion (17). However, in order to accomplish this, a careful application of sEMG decomposition and expertise in signal acquisition, interpretation of results, and manual assessment of decomposition quality is required (14-16). Further, the examination of the Motor Unit Number Index (MUNIX) in paralyzed muscles has been implemented to monitor $\mathrm{MU}$ loss after SCI (18). However, this approach requires intense experimental and computational setup, and specific selection criteria which may not be clinically feasible.

\section{Lack of Available Time for a Clinician}

A study collected perspectives of 22 clinicians [physical therapists (PT), occupational therapists (OT), and physiatrists] and reported limited clinician time as one of the barriers to the uptake of sEMG technology in clinics (11). The time-consuming aspect of sEMG technology presents a significant barrier to its translation into clinical practices. Electrodes and skin preparations, electrode placements, equipment setup, collecting maximal volitional contractions (MVC) for normalization prior to recording the data during activities of interest take significant time. Figure 1 illustrates the sEMG placements for recording of lower extremity responses from an individual with an SCI. 


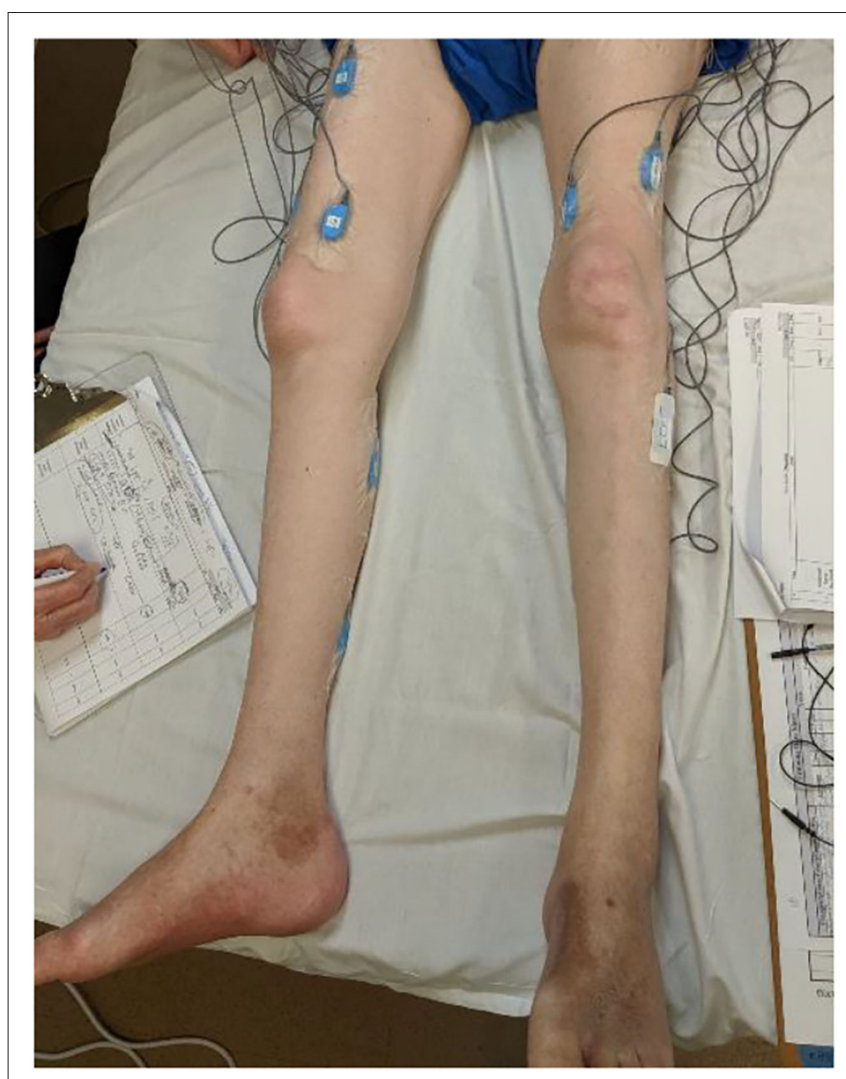

FIGURE 1 | An EMG set up for assessing neuromuscular responses prior to a rehabilitation intervention for an individual with $\mathrm{SCl}$.

Balancing a busy work schedule has been reported as one of the barriers to caring for patients, particularly for novice PTs (19). Therefore, the acceptance of sEMG technology that requires significant prep-time is low as the added time could adversely affect PT performance and care in the clinic.

\section{Limited Background and Training Through Professional Curricula}

Most of the PT and OT programs offer wide-ranging coursework in the rehabilitation domain including human anatomy, neuroscience, biomechanics, kinesiology, movement analysis, evidence-based practice, pharmacological interventions, etc. Irrespective of the breadth of topics covered, there is minimal focus on the technological aspects of rehabilitation. As a result, rehabilitation tools such as sEMG are theoretically taught, but practical knowledge imparted is limited. Further, the educational content may not cover ever-evolving aspects of sEMG technology and its applications. Feldner et al. reported that many clinicians felt less confident to use sEMG in clinics due to their limited experience (11). According to the study, newer clinicians pointed the "need for practice" and the seasoned clinicians weren't "tech savvy," making the clinical adoption of sEMG technology difficult (11). However, one limitation of this survey was the limited geographical spread of the clinicians who participated as they were recruited from rehabilitation settings within the Seattle metropolitan area (WA, USA). In a more recent survey by Manca et al., 35 EMG experts from different educational, professional and geographical backgrounds supported the clinical utility of sEMG for optimizing the quantification of muscle and physical function, to define the intervention plan, and optimize other methods used to quantify muscle and physical function (20). However, the collective opinion of these experts also confirmed the utilization of sEMG was more common in technical/methodological research than clinical research (20). The barriers that prevent prompt transfer of sEMG into practice were reported to be slow dissemination of research findings and the lack of education on sEMG (20). Further, successful adoption of any technology in the clinic not only involves collecting the information/data but also helps in making data-driven clinical decisions in functional diagnosis, recommending appropriate interventions, and optimizing the rehabilitation outcomes. In terms of sEMG, the processing and interpretation of the data require a multidisciplinary approach. This involves the working knowledge of several technical domains such as instrumentation, signal processing and analysis, algorithm development, and statistical analyses. The availability of such expertise can be challenging in a clinical setting. Identifying the experts with such a skillset and establishing collaborations could be timeconsuming, and impractical for daily-workflow at the clinic. If a clinician wants to gain the necessary working knowledge on sEMG technology, there is no centralized knowledge-base where clinicians can, not only develop their understanding of sEMG procedures and data analyses but also interact with other clinicians and researchers in this specific domain to share ideas, discuss outcomes and even collaborate at the institutional levels. The training and education of teachers who are educating future clinicians is another important factor. In many countries where there are no doctoral-level programs in rehabilitation or physiotherapy, there is a scarcity of academic professors with doctorate-level credentials. Therefore, the educational experience of students in such countries may lack rigor, practical exposure to the technology, and the state-of-the-art information on sEMG practices and guidelines.

\section{Lack of Technology Transfer From Research to Clinic}

The field of sEMG is always evolving and new algorithms for sEMG processing, analysis, and classifications are continuously being developed. However, the rate at which these technological advances are frequently integrated into the existing sEMG systems is limited. For instance, many of the existing offthe-shelf sEMG systems have not gone beyond implementing the basic sEMG features such as mean and root-mean-square (RMS) amplitudes, moving average or RMS envelopes, basic filtering and rectifications, and basic Fourier-based analysis. Automatic burst or ON-OFF detections, activation timing analyses, signal decomposition, and time-frequency analyses are widely published (21-25) and accepted EMG analysis techniques that have not been integrated into most of the commercial systems; as a result, these techniques have not been transferred from research to clinic. This issue stems from lack of education or training on the application of such analysis methods in a clinical setting, resulting in virtually no demand for a commercial 
EMG system with these capabilities, which in turn creates an insignificant market to manufacture such EMG devices. Therefore, the absence of commercial pressure further limits the development of said devices and education of operators to ultimately transfer research findings into the clinic.

\section{Institutional Level Barriers}

In addition to the sEMG setup time, other challenges hinder the adoption of sEMG technology in the clinic. Such barriers include the functionality in multiple environments, portability, the facility layout, purchasing cost and maintenance, providing evidence to support returns on such investments, and staff training.

\section{Barriers Specific to the SCI Population}

The need for assessing neuromuscular responses is highly significant for individuals with SCI, particularly motor complete SCI (cSCI). Studies have demonstrated the presence of intact neuronal axons across the lesion, even after cSCI (17). For instance, Calancie et al. (26) reported retained voluntary EMG control over one muscle in the foot in a small group of participants classified as motor complete. These findings highlight the significance of the ability to monitor neuromuscular responses during neuromuscular electrical stimulation (NMES) for cSCI for whom any functional and motor-related changes may not be apparent, while intrinsic electrophysiological changes and residual volitional neuromuscular drive may still be present. In evaluating the efficacy of any clinical therapy, the effects may not be visible at the functional or biomechanical levels but changes could be present at the neuromuscular level. Therefore, assessing neuromuscular output is critical to optimize the effects of any rehabilitation intervention for SCI. Currently, there are no standardized procedures for processing and interpreting sEMG data specific to the cSCI population; this may have vastly contributed to the diverse sEMG interpretations and/or continued reliance on outcome measures, such as force and torque. In addition, the lack of standards for sensors, configurations, electrode placement, and recording protocols has adversely affected the possibility of its integration into routine clinical use (9). Despite the 20 -year presence of the EU project on "Surface EMG for Non-Invasive Assessment of Muscles (SENIAM)," real international standards are still missing (10). The diminished or weaker sEMG signals yield limited consensus on answers to the most basic questions such as, "is the muscle active?" "what is the strength of the activation?" or more complicated ones such as, "what is the volitional contribution and how it relates to the applied stimuli during electrically induced activations?" Answers to such questions remain unclear as there is no standardized approach to first process and then interpret such data. The existing off-theshelf systems are not specifically tuned to address these SCIspecific challenges. For example, the most significant barrier in using sEMG during FES is interpreting the recorded sEMG signals due to the overpowering presence of stimulation artifact. The stimulation artifact is a broadband signal with widespread stimulation frequency harmonics at high amplitudes that engulf the myoelectric responses in sEMG. Particularly when a train of ES pulses is applied, the sEMG recordings are accompanied by
ES artifact spikes with magnitudes that are manifold compared to the actual MU outputs. Moreover, the presence of stimulation artifact is not confined in the time-domain; it is also observed in the frequency domain. The harmonics of stimulation frequency overlap with the majority of the energy bands in a typical sEMG frequency spectrum $(20-350 \mathrm{~Hz})$. As a result, traditional selected-filtering of frequency bands, to remove ES artifacts, is ineffective and results in significant data loss (23). The ES artifact affects features derived from the sEMG signal; for instance, it biases conduction velocity estimations, spectral characteristic frequencies, and M-wave amplitudes (27). In the domain of SCI rehabilitation, where ES waveforms are often delivered as bursts (train of pulses) with high intensities and wide-ranging frequencies, the resultant contamination of sEMG recordings obstructs the understanding of the direct implication of FES on the neuromuscular output in terms of activation intensity (voluntary or ES induced), MU recruitment, and muscle fatigue. This is particularly impeding in studies where FES is combined with volitional efforts that need to be monitored or modulated in real-time to achieve optimal outcomes.

\section{FUTURE DIRECTIONS}

Rehabilitation professionals' acceptance and adoption of technologies rely on conditions that facilitate their use such as scheduling, support and a conductive environment (28). The following are the steps toward achieving these key aspects of sEMG utilization in the clinical neurorehabilitation.

\section{Enhancing Knowledge and User-Experience}

In order to ensure all rehabilitation professionals, especially clinicians, get an early exposure to the sEMG technology, the educational and professional training programs could integrate hands-on sEMG experience through case studies or small research projects. The clinicians could also enhance their involvement in ongoing sEMG-related research activities and get exposed to the several practical aspects of sEMG through interactions with their non-clinical counterparts (e.g., engineers, technicians, data scientists). The interfaces running the EMG data collection and processing algorithms with minimal user inputs could be beneficial for their widespread implementations. Another goal could be set to successfully transfer EMG-related research products (data collection, processing and analysis algorithms) into a clinical environment. Irrespective of the programming platforms (Matlab, Python, etc.) on which these algorithms are built upon, simple user-interfaces, application programming interfaces (APIs) and/or open-source executables can be created for their unobstructive and intuitive use by the clinicians with non-technical backgrounds. A centralized knowledge-base can be used to create and disseminate the sEMG tutorials on topics ranging from the basics of sEMG technology to step-by-step guidelines for data processing. Such a centralized open-source platform can also facilitate the collaborations among investigators and sEMG users with overlapping interests. With the help of well-established societies such as International Society of Electromyography and Kinesiology (ISEK), IEEE 
Engineering in Medicine and Biology Society (EMBS), Society for Neuroscience (SFN), and several societies of clinical motion analysis [Gait Clinical Movement Analysis Society (GCMAS), the European Society for Movement Analysis in Adults and Children (ESMAC), Societa' Italiana di Analisi del Movimento in Clinica (SIAMOC) etc.], the long-term goal can be set to developing international scientific meetings or chapters specific to sEMG applications in specific rehabilitation domain (e.g., FES) where the specific pool of researchers can meet, share knowledge and collaborate. In recent years, efforts have been made to provide open-access tutorials and consensus articles on sEMG-related best practices, such as the consensus standards and guidelines on the sEMG detection (29), sEMG signal conditioning and preprocessing (30), and analysis of MU discharge characteristics using HDEMG (14). The Consensus for Experimental Design in Electromyography (CEDE) project, an international initiative which aims to guide decision-making in recording, analysis, and interpretation of sEMG have published the guidelines on the sEMG electrode selection and amplitude normalization $(31,32)$. Despite of these past and present efforts, these wellaccepted guidelines, procedures and standards are not known to many clinicians. The paradigm shift in transferring such significant knowledge to clinic is only possible when the new generations of students pursuing education and professional training in clinical rehabilitation (e.g., PT, PTA, MPT, DPT, $\mathrm{DScPT}, \mathrm{PhD}$ ) are taught these "best practices in $\mathrm{sEMG"} \mathrm{by}$ qualified teachers.

\section{EMG for Real-Time Monitoring and Biofeedback During Rehabilitation}

The instantaneous quantification of muscle response can serve as an important marker to track the impairment as well as recovery during rehabilitation. With access to the EMG in real-time, the clinicians or researchers can quantify, track, and manipulate levels of voluntary efforts by modulating intervention parameters. For example, if a clinician observes that the FES frequency of $100 \mathrm{~Hz}$ is causing a muscle to fatigue faster with less voluntary participation (shown by EMG features such as amplitude), s(he) could change to a lower stimulation frequency, which could potentially increase voluntary contribution and reduce fatigue due to stimulation, thus making the session still productive. Such modulations could happen simply by patient's own feedback on fatigue but the data-driven nature of this decision making could make the training more objective, patientspecific, safe and less ad hoc. This could result in more effective interventions for better long-term benefits.

\section{A Ranking System for Standardization of EMG Interpretations for the SCI}

Motivated by the ranking system provided by Heald et al. (17), a standardized sEMG ranking system can be developed to quantify the state of the residual neuromuscular output, especially during FES-based rehabilitation for SCI. For example, Rank 1 - sEMG signal can be classified as no activity, baseline noise; Rank 2 - Sparse MU action potentials; Rank 3 - Burst of activity but no clear correlation to stimulation profile (e.g., FES, etc.); Rank 4 - Burst of activity with partial correlation to stimulation;
Rank 5 - Repeated burst of visible activity that is significantly correlated with applied stimulation. Ranking procedures can be validated by visual inspection as well as automated, softwaredriven inspections. Such a standardized approach can track progress during or after different interventions. Once accepted and implemented, common standardized outcomes would enable comparing different interventions for efficacy.

\section{The Potential Impact on the Rehabilitation Costs for SCl}

For many of the SCI patients, functional or motor changes may not be present but electrophysiological changes or residual voluntary muscle activations may still be present $(17,26,33$, 34). If a clinician cannot directly track the volitional efforts or functional improvements, then medical reimbursement is suspended after only a few weeks with no ultimate benefit to the participant. If sEMGs show the neuromuscular changes during an intervention for individuals with SCI with no changes in functional status, researchers and clinicians can still continue with ongoing interventions and anticipate better outcomes. On the other hand, investing in expensive interventions for several months for non-responders is a financial liability. Thus, sensitive and reliable measures of neuromuscular recovery, designed specifically for the spectrum of SCI-induced deficits can lead to long-term functional improvement that would have a dramatic impact both on the quality of life and financial liability for those suffering from SCI.

In summary, addressing the current barriers in widespread use of sEMG in SCI rehabilitation will require a collaborative, interdisciplinary, and unified approach. Nonetheless, sEMG technology has the potential to present significant opportunities that can allow clinicians and researchers to transform future interventions into effective and impactful rehabilitation modalities for individuals with SCI.

\section{DATA AVAILABILITY STATEMENT}

The data sharing will be contingent upon the regulations applied by the funding agency. Requests to access the datasets should be directed to rpilkar@kesslerfoundation.org.

\section{ETHICS STATEMENT}

The studies involving human participants were reviewed and approved by Kessler Foundation Institutional Review Board. The patients/participants provided their written informed consent to participate in this study.

\section{AUTHOR CONTRIBUTIONS}

$\mathrm{RP}$ and KM drafted the manuscript. All authors contributed to the revisions.

\section{FUNDING}

This work was supported by the New Jersey Commission on Spinal Cord Research grants (CSCR20ERG013, 314 CSCR14ERG007) and Kessler Foundation. 


\section{REFERENCES}

1. Musselman KE, Shah M, Zariffa J. Rehabilitation technologies and interventions for individuals with spinal cord injury: translational potential of current trends. J Neuroeng Rehabil. (2018) 15:40. doi: 10.1186/s12984-018-0386-7

2. Luo S, Xu H, Zuo Y, Liu X, All AH. A review of functional electrical stimulation treatment in spinal cord injury. Neuromolecular Med. (2020) 22:447-63. doi: 10.1007/s12017-019-08589-9

3. Alajam R, Alqahtani AS, Liu W. Effect of body weight-supported treadmill training on cardiovascular and pulmonary function in people with spinal cord injury: a systematic review. Top Spinal Cord Inj Rehabil. (2019) 25:35569. doi: 10.1310/sci2504-355

4. Ho CH, Triolo RJ, Elias AL, Kilgore KL, DiMarco AF, Bogie K, et al. Functional electrical stimulation and spinal cord injury. Phys Med Rehabil Clin N Am. (2014) 25:631-54, ix. doi: 10.1016/j.pmr.2014.05.001

5. Ragnarsson KT. Functional electrical stimulation after spinal cord injury: current use, therapeutic effects and future directions. Spinal Cord. (2008) 46:255-74. doi: 10.1038/sj.sc.3102091

6. Marino RJ, Barros T, Biering-Sorensen F, Burns SP, Donovan WH, Graves DE, et al. International standards for neurological classification of spinal cord injury. J Spinal Cord Med. (2003) 26 (Suppl. 1):S506. doi: 10.1080/10790268.2003.11754575

7. Peckham P, Knutson J. Functional electrical stimulations for neuromuscular applications. Ann Rev Biomed Eng. (2005) 7:327-60. doi: 10.1146/annurev.bioeng.6.040803.140103

8. Merletti R, Farina D. Surface Electromyography: Physiology, Engineering, and Applications. Hoboken, NJ: Wiley-IEEE Press (2016).

9. Hogrel JY. Clinical applications of surface electromyography in neuromuscular disorders. Neurophysiol Clin. (2005) 35:5971. doi: 10.1016/j.neucli.2005.03.001

10. Campanini I, Disselhorst-Klug C, Rymer WZ, Merletti R. Surface EMG in clinical assessment and neurorehabilitation: barriers limiting its use. Front Neurol. (2020) 11:934. doi: 10.3389/fneur.2020.00934

11. Feldner HA, Howell D, Kelly VE, McCoy SW, Steele KM. "Look, Your Muscles Are Firing!": a qualitative study of clinician perspectives on the use of surface electromyography in neurorehabilitation. Arch Phys Med Rehabil. (2019) 100:663-75. doi: 10.1016/j.apmr.2018.09.120

12. Pullman SL, Goodin DS, Marquinez AI, Tabbal S, Rubin M. Clinical utility of surface EMG: report of the therapeutics and technology assessment subcommittee of the American Academy of Neurology. Neurology. (2000) 55:171-7. doi: 10.1212/WNL.55.2.171

13. Drost G, Stegeman DF, van Engelen BG, Zwarts MJ. Clinical applications of high-density surface EMG: a systematic review. J Electromyogr Kinesiol. (2006) 16:586-602. doi: 10.1016/j.jelekin.2006.09.005

14. Del Vecchio A, Holobar A, Falla D, Felici F, Enoka RM, Farina D. Tutorial: analysis of motor unit discharge characteristics from high-density surface EMG signals. J Electromyogr Kinesiol. (2020) 53:102426. doi: 10.1016/j.jelekin.2020.102426

15. Farina D, Holobar A, Merletti R, Enoka RM. Decoding the neural drive to muscles from the surface electromyogram. Clin Neurophysiol. (2010) 121:1616-23. doi: 10.1016/j.clinph.2009.10.040

16. Nawab SH, Chang SS, De Luca CJ. High-yield decomposition of surface EMG signals. Clin Neurophysiol. (2010) 121:160215. doi: 10.1016/j.clinph.2009.11.092

17. Heald E, Hart R, Kilgore K, Peckham PH. Characterization of volitional electromyographic signals in the lower extremity after motor complete spinal cord injury. Neurorehabil Neural Repair. (2017) 31:583-91. doi: 10.1177/1545968317704904

18. Li X, Jahanmiri-Nezhad F, Rymer WZ, Zhou P. An Examination of the Motor Unit Number Index (MUNIX) in muscles paralyzed by spinal cord injury. IEEE Trans Inf Technol Biomed. (2012) 16:11439. doi: 10.1109/TITB.2012.2193410

19. Greenfield BH, Anderson A, Cox B, Tanner MC. Meaning of caring to 7 novice physical therapists during their first year of clinical practice. Phys Ther. (2008) 88:1154-66. doi: 10.2522/ptj.20070339
20. Manca A, Cereatti A, Bar-On L, Botter A, Della Croce U, Knaflitz $M$, et al. A survey on the use and barriers of surface electromyography in neurorehabilitation. Front Neurol. (2020) 11:573616. doi: 10.3389/fneur.2020.573616

21. Pilkar R, Ramanujam A, Nolan KJ. Alterations in spectral attributes of surface electromyograms after utilization of a foot drop stimulator during post-stroke gait. Front Neurol. (2017) 8:449. doi: 10.3389/fneur.2017.00449

22. Pilkar R, Yarossi M, Nolan KJ. EMG of the tibialis anterior demonstrates a training effect after utilization of a foot drop stimulator. NeuroRehabilitation. (2014) 35:299-305. doi: 10.3233/NRE-141126

23. Pilkar R, Yarossi M, Ramanujam A, Rajagopalan V, Bayram MB, Mitchell M, et al. Application of empirical mode decomposition combined with notch filtering for interpretation of surface electromyograms during functional electrical stimulation. IEEE Transact Neural Syst Rehabil Eng. (2017) 25:126877. doi: 10.1109/TNSRE.2016.2624763

24. Solnik S, Rider P, Steinweg K, DeVita P, Hortobagyi T. Teager-Kaiser energy operator signal conditioning improves EMG onset detection. Eur J Appl Physiol. (2010) 110:489-98. doi: 10.1007/s00421-010-1521-8

25. Zhang $X$, Zhou $P$. Filtering of surface EMG using ensemble empirical mode decomposition. Med Eng Phys. (2013) 35:53742. doi: 10.1016/j.medengphy.2012.10.009

26. Calancie B, Molano MR, Broton JG. Abductor hallucis for monitoring lowerlimb recovery after spinal cord injury in man. Spinal Cord. (2004) 42:57380. doi: $10.1038 /$ sj.sc. 3101640

27. Mandrile F, Farina D, Pozzo M, Merletti R. Stimulation artifact in surface EMG signal: effect of the stimulation waveform, detection system, and current amplitude using hybrid stimulation technique. IEEE Trans Neural Syst Rehabil Eng. (2003) 11:407-15. doi: 10.1109/TNSRE.2003.819791

28. Liu L, Miguel Cruz A, Rios Rincon A, Buttar V, Ranson Q, Goertzen D. What factors determine therapists' acceptance of new technologies for rehabilitation - a study using the Unified Theory of Acceptance and Use of Technology (UTAUT). Disabil Rehabil. (2015) 37:44755. doi: 10.3109/09638288.2014.923529

29. Merletti R, Muceli S. Tutorial. Surface EMG detection in space and time: Best practices. J Electromyogr Kinesiol. (2019) 49:102363. doi: 10.1016/j.jelekin.2019.102363

30. Merletti R, Cerone GL. Tutorial. Surface EMG detection, conditioning and pre-processing: best practices. J Electromyogr Kinesiol. (2020) 54:102440. doi: 10.1016/j.jelekin.2020.102440

31. Besomi M, Hodges PW, Clancy EA, Van Dieën J, Hug F, Lowery M, et al. Consensus for experimental design in electromyography (CEDE) project: amplitude normalization matrix. J Electromyogr Kinesiol. (2020) 53:102438. doi: 10.1016/j.jelekin.2020.102438

32. Besomi M, Hodges PW, Van Dieën J, Carson RG, Clancy EA, DisselhorstKlug C, et al. Consensus for experimental design in electromyography (CEDE) project: electrode selection matrix. J Electromyogr Kinesiol. (2019) 48:12844. doi: 10.1016/j.jelekin.2019.07.008

33. Dimitrijevic MR, Faganel J, Lehmkuhl D, Sherwood A. Motor control in man after partial or complete spinal cord injury. Adv Neurol. (1983) 39:915-26.

34. McKay WB, Lim HK, Priebe MM, Stokic DS, Sherwood AM. Clinical neurophysiological assessment of residual motor control in post-spinal cord injury paralysis. Neurorehabil Neural Repair. (2004) 18:144-53. doi: 10.1177/088843900426 7674

Conflict of Interest: The authors declare that the research was conducted in the absence of any commercial or financial relationships that could be construed as a potential conflict of interest.

Copyright (c) 2020 Pilkar, Momeni, Ramanujam, Ravi, Garbarini and Forrest. This is an open-access article distributed under the terms of the Creative Commons Attribution License (CC BY). The use, distribution or reproduction in other forums is permitted, provided the original author(s) and the copyright owner(s) are credited and that the original publication in this journal is cited, in accordance with accepted academic practice. No use, distribution or reproduction is permitted which does not comply with these terms. 